\title{
La interculturalidad como macrocompetencia en la enseñanza de lenguas extranjeras: revisión bibliográfica y conceptual
}

\author{
Benamí Barros García y Galina M. Kharnásova \\ Universidad de Granada
}

Recibido: 3 de marzo de 2010 / Aceptado: 15 de septiembre de 2011

ISSN: $1697-7467$

\begin{abstract}
RESUMEN: El presente artículo se centra en el estudio de la interculturalidad en tanto que factor esencial y necesario en la enseñanza y adquisición de lenguas extranjeras. Para ello se ha optado por partir de una revisión de la definición de competencia intercultural, así como de su consolidación terminológica, con el fin de, finalmente, justificar la idoneidad y posibilidades prácticas de un enfoque que conciba la competencia intercultural como macrocompetencia. Asimismo, serán parte central del presente estudio las condiciones para una óptima transversalización, integración y desarrollo de la competencia intercultural en el aprendiente.

Palabras clave: Interculturalidad, macrocompetencia, enseñanza y adquisición de LE, lingüística aplicada.
\end{abstract}

Interculturality as Macrocompetence in Foreign Language Teaching: Conceptual and Bibliographical Overview

\begin{abstract}
This article focuses in the study of interculturality as an essential and necessary factor in foreign language learning/acquisition. To begin we review the definition of intercultural competence along with the terminological validity, so we can justify the definitions suitability and the possibilities posed by an approach that conceives intercultural competence as a macrocompetence. We will also deal with those conditions needed for achieving optimal levels of mainstreaming, integration and formation of intercultural competence in foreign language learners.

Keywords: Interculturality, macrocompetence, foreign language learning/acquisition, applied linguistics.
\end{abstract}

\section{Desde la COMPeTenCia COMUNiCATIVA HACIA UNA CONCEPCIÓN INTER-CUL-}

\section{TURAL DE LA COMUNICACIÓN}

Las investigaciones que propusieron y desarrollaron el concepto de competencia intercultural dentro de la lingüística y otras áreas interrelacionadas con ésta, conviene recordar, tuvieron lugar en un marco geopolítico, etnográfico y científico altamente propicio (y determinante): por un lado, las investigaciones lingüísticas, una vez superada la 
focalización frástica ${ }^{1}$ de los estudios lingüísticos y literarios, derivaron progresivamente en un interés por el receptor hasta consolidarse en diversos paradigmas que, a la larga, iban a influir sustancialmente en el desarrollo de la disciplina de la enseñanza y adquisición de lenguas extranjeras; por otro, la multiculturalidad era un hecho social generalizado y ya insoslayable para cualquier disciplina ${ }^{2}$. Entre los paradigmas lingüísticos y ramas que facilitaron la consolidación de una base para la reformulación de las teorías de enseñanza y aprendizaje de lenguas extranjeras desde una perspectiva, primero, comunicativa y, después, pragmática, podemos destacar las siguientes: Pragmática, Lingüística del Texto, Estética de la Recepción, Semiótica, CDA, Etnografía de la Comunicación, etc. Precisamente en la última de las citadas se fragua el concepto central de esta investigación: la competencia intercultural, concepto derivado de la consideración de que toda cultura está configurada por una serie de elementos comunicativos que pueden ser analizados (Hall, 1959).

A esto hay que sumar el carácter multidisciplinar que gobernaba en la segunda mitad del siglo XX las investigaciones lingüísticas y que propició un aperturismo en cuanto a enfoques metodológicos de estudio y análisis; algo que indudablemente proporcionó numerosas herramientas novedosas y modelos interpretativos para el análisis de fenómenos que hasta entonces parecían tener difícil resolución desde una perspectiva lingüística clásica ${ }^{3}$. Parece claro por qué los conceptos manejados por estas disciplinas hasta entonces tuvieron que ser, casi forzosamente, reformulados. Términos como comunicación, lector, oyente, código, discurso, lengua y cultura pasaron a estar en el punto de mira de una nueva terminología ${ }^{4}$ que hacía uso de diferentes ramas del saber para indagar en los procesos comunicativos. Para el tema que aquí nos ocupa, sin duda los más relevantes son los cambios introducidos en la concepción de cultura y comunicación dentro de los clásicos binomios lengua-cultura, lengua-comunicación, de cuyo análisis no nos ocuparemos aquí, limitándonos simplemente a poner de manifiesto la tendencia hacia un estudio más profundo de la comunicación en relación con la cultura desde diferentes vertientes ${ }^{5}$.

La preeminencia de un enfoque centrado en la comunicación como fenómeno social propició que el uso pasara a un primer plano tanto en las investigaciones de lingüística aplicada como en la práctica metodológica de enseñanza de lenguas extranjeras. Desde el modelo de Hymes (1972), según el cual en todo acto comunicativo intervienen la

1 Véase Dubois (1994: 294).

2 Para una revisión de los argumentos que justifican la multiculturalidad de la sociedad actual en términos políticos, demográficos y económicos, véase Bartolomé et al. (2002).

${ }^{3}$ No quiere decir esto que a día de hoy esté totalmente aceptada la multidisciplinariedad de la enseñanza y aprendizaje de lenguas extranjeras, puesto que el debate sigue estando presente en prácticamente todas las áreas implicadas. La dificultad metodológica que implica el hecho de proponer modelos mixtos puede ilustrarse con el caso de la Psicolingüística, en la que, como indica Javier Zanón (2007), existe un espectro de aceptación que se mueve entre dos extremos: autores totalmente contrarios a la interrelación con la Metodología de enseñanza de lenguas (McLaughlin, 1987 y Pienemann, 1984 - éste de corte más moderado -) y otros que niegan cualquier posible refutación a tal vinculación (Krashen y Terrell, 1983).

4 Debemos reconocer que a día de hoy sigue siendo, en muchos casos, confusa.

5 Entre otras fuentes, véase Wierzbicka (1997, 1999). 
situación, los participantes, los fines, las sucesiones de actos, los códigos o claves, la instrumentación, las normas y el género, parece claro el desplazamiento hacia la concepción social de la comunicación y su correspondiente aplicación al concepto de aprendiente. De hecho, son objeto de estudio las normas de interpretación derivadas del uso de la lengua (la actuación lingüística chomskiana), según la hipótesis de que el hablante ya no sólo habla, sino que también comunica ${ }^{6}$. Estas normas no tardaron en demostrar que, dentro de una ya amplia tradición lingüística en los estudios sobre Pragmática y Filosofía del Lenguaje, la visión del mundo es un factor determinante y diferenciador durante el proceso de interpretación de un discurso. Y si lo es en la interpretación también debe ser relevante a la hora de adquirir y enseñar una lengua extranjera, puesto que la eficacia comunicativa estará siempre condicionada por la aceptabilidad y apropiación.

Así las cosas, la competencia comunicativa sustituye o, mejor dicho, engloba dentro de sí la competencia lingüística o gramatical (en el argot generativista). No es, por tanto, casual que Hymes (1971) critique la insuficiencia de ésta en tanto que no tiene en cuenta los factores socioculturales implicados en el contexto conversacional. De hecho, para paliar esta carencia Hymes (op.cit.) propone una serie de criterios para determinar el grado de apropiación y aceptabilidad de un enunciado, dependiendo de si es o no posible, factible, apropiado y real; criterios éstos que bien pueden ser considerados como base de las posteriores formulaciones del concepto de competencia comunicativa. Así, lo relevante de esta propuesta, además de su carácter innovador, es el hecho de dejar entrever que tras cada uno de los criterios hay toda una red de competencias que el aprendiente deberá adquirir. Precisamente en esta línea y con esta finalidad se postulan varios modelos, orientados hacia la contextualización de la competencia comunicativa dentro del estudio de la enseñanza y aprendizaje de lenguas extranjeras, y que dan fe de la importante evolución que ha experimentado el concepto de competencia comunicativa (entre otros, Canale y Swain, 1980; van Ek, 1986; Bachman, 1990; Celce-Murcia, Dörnyei y Thurrell, 1995).

Además de la incuestionable evolución del concepto de competencia comunicativa ${ }^{7}$, es necesario advertir que, si bien no con tanta eficacia como debiera y nos gustaría, estos modelos y otras investigaciones relativas a la metodología de enseñanza y aprendizaje de lenguas extranjeras han propiciado que la nueva concepción del aprendiente de una lengua extranjera, entendido ya no como hablante ideal de una lengua como no nativo, sino como partícipe activo y real de un proceso social comunicativo ${ }^{8}$, influya en prácticamente todos los niveles y estadios de la enseñanza: desde la creación de materiales

${ }^{6}$ Véase Gumperz (1982).

7 Nótese que entre los aspectos que han despertado mayor interés destacan la competencia sociolingüística y la competencia pragmática. En particular, la competencia pragmática (Celce-Murcia, Dornyei y Thurrell 1995; Cenoz y Valencia, 1996; Kasper y Rose, 2001) en cuanto al estudio y análisis de errores, malentendidos y conductas conversacionales inapropiadas, debido a que, a diferencia de los errores gramaticales, pueden condicionar la percepción e imagen que el interlocutor se forme del aprendiente. Las consecuencias de una competencia pragmática mal configurada o desarrollada (Gómez Morón, 2004) pueden ser nefastas, siendo aún más determinantes en los usos de las lenguas de especialidad.

${ }^{8}$ Siguiendo a Kramsch (1998: 23-37), utilizaremos el término "hablante intercultural". 
más efectivos para la adquisición de la LE hasta la ampliación de posibilidades del aula como espacio educativo.

En general, y gracias a los avances en los estudios de antropología dedicados a la enseñanza de lenguas extranjeras, la atención de los nuevos métodos se centra en el desarrollo por parte del aprendiente del comportamiento conversacional apropiado en la lengua extranjera. Ya partamos del esquema de Scheflen (1984) sobre el comportamiento comunicativo, ya lo hagamos desde la Triple Estructura Básica de la Comunicación de Poyatos (1994, I: 130), quien diferencia en el comportamiento comunicativo entre el verbal-lingüístico, el paralenguaje y el no verbal, todo indica a que la definición de comunicación (no sólo en una lengua extranjera) debe realizarse en términos de eficacia y no de corrección gramatical ${ }^{9}$. Para que una comunicación sea eficaz (y en los nuevos enfoques sólo se entenderá como comunicación aquella que lo sea) Woods (1998: 39) propone distinguir seis procesos fundamentales:

* Percepción y comprensión

* Participación en la comunicación verbal

* Participación en la comunicación no verbal

* Muestra de interés

* Creación de una atmósfera óptima para interactuar

* Adaptación al contexto

Como veremos, esta clasificación de criterios está estrechamente relacionada con la concepción de competencia intercultural, hasta tal punto de que podemos presuponer que Woods (loc.cit.) en realidad sintetiza los requisitos necesarios para que una comunicación sea intercultural.

\section{LA COMPETENCIA INTERCULTURAL COMO MACROCOMPETENCIA}

Llegados a este punto, podemos plantearnos como tarea analizar en qué grado influye en lo expuesto hasta el momento el hecho de concebir la comunicación en términos de interculturalidad.

En cualquier caso, y aun teniendo en cuenta que durante la última década los estudios interculturales han experimentado un auge espectacular, entre otras razones por la imperiosa necesidad de estudiar un fenómeno social de tamaño calibre, creemos necesaria una aclaración terminológica. El hecho de que hasta la fecha no podamos hablar de la existencia de un consenso terminológico en los estudios interculturales es bastante significativo, a la vez que preocupante ${ }^{10}$. El problema, aparte de los conflictos geopolíticos subyacentes en casi cualquier ejercicio terminológico que ataña al ámbito internacional,

\footnotetext{
9 Veremos que la eficacia servirá de base para la comprensión de lo que se entiende por comunicación intercultural.

10 Véase al respecto Alsina (1997).
} 
radica en la utilización de diferentes conceptos para referirse supuestamente a un mismo fenómeno: interculturalidad, multiculturalidad, pluriculturalidad, etcétera ${ }^{11}$.

Aquí nos serviremos de la premisa de que la interculturalidad hace referencia a las (inter)relaciones que ocurren en un espacio común (por ejemplo, el aula), en tanto que contexto multicultural ${ }^{12}$. Por tanto, diremos que la interculturalidad parte de una conceptualización dinámica del contexto multicultural, es decir, de las relaciones que en éste pueden o no tener lugar. Es precisamente este hecho de importancia capital para comprender que no todo lo que ocurra en un espacio o contexto multicultural ha de ser etiquetado como intercultural ad hoc. La multiculturalidad, en tanto que existencia como tal de diversas culturas en un mismo contexto espacial y temporal, puede definirse con verbos de existencia y ser empíricamente comprobada y baremada. Por su parte, y frente al estatismo por definición de la multiculturalidad, lo intercultural refiere una realidad en su dinámica de continua configuración, en tanto que entramado social de sujetos interactuantes. Requiere, pues, partir de una idea de cultura como proceso, como sistema en evolución ${ }^{13}$. Mientras que la multiculturalidad es un hecho, cuyo reconocimiento, aprobación, desaprobación o valoración sólo puede ser realizada mediante estrategias que impliquen interacción, esto es, mediante estrategias interculturales, la interculturalidad podría identificarse con una predisposición a nivel profundo de la conciencia de los sujetos, de las sociedades o, siguiendo a Alsina (Rodrigo Alsina, 1997), de las comunidades culturales.

Esta misma diferenciación terminológica cobra gran importancia en el ámbito de la enseñanza y aprendizaje de lenguas extranjeras, en que perdura otro de los dilemas terminológicos clave dentro de los estudios interculturales y que, en los últimos años, viene situándose en el foco de atención de gran parte de los modelos educativos propuestos desde una perspectiva intercultural. Nos referimos a la distinción realizada por, entre otros, Beacco y Byram (2007) en cuanto al plurilingüismo, en tanto que competencia, y el multilingüismo, como indicador de coexistencia de más de una lengua en un mismo espacio. Cuestión esta sobre la que volveremos a incidir más adelante.

Recordemos ahora que, si bien la realidad es multicultural (Israel 1995: 63) y, consecuentemente, los aprendientes viven en un espacio multicultural, las relaciones que se produzcan dentro de ese espacio habrán de cumplir una serie de criterios para poder ser tildadas de interculturales.

Ante un contexto multicultural ya difícilmente disimulable, parece consensuada la propuesta de considerar necesaria una competencia intercultural en el aprendiente ${ }^{14}$. Tal reflexión está presente en el Marco común europeo de referencia para las lenguas: aprendizaje, enseñanza, evaluación (en lo sucesivo, MCERL), aunque creemos que su

${ }^{11}$ No entraremos a debatir la terminología anglosajona ni las equivalencias conceptuales con los términos españoles.

12 Para una explicación estadística de la presencia de diferentes culturas en las aulas, se pueden consultar los Datos Avance que publica el Ministerio de Educación para los diferentes cursos académicos. Aunque los datos son en sí reveladores, añadiremos que en algunos centros públicos de determinadas localidades durante los cursos académicos 2009/10 y 2010/11 se ha llegado a superar el $30 \%$ de alumnado extranjero.

13 Idea explotada por las teorías sistémicas y la semiótica.

14 Según Byram (1997) esta competencia nace del modelo de Van Ek (1986). 
presencia adolece, en parte, de una explicación fundamentada del verdadero papel que ocupa en el proceso de enseñanza y aprendizaje de lenguas extranjeras.

En el MCERL, la competencia intercultural no aparece recogida como tal, sino como una serie de elementos que, entendemos, son constituyentes de aquélla. En el capítulo dedicado a las competencias generales (5.1), se habla de la consciencia intercultural:

El conocimiento, la percepción y la comprensión de la relación entre «el mundo de origen» y «el mundo de la comunidad objeto de estudio» (similitudes y diferencias distintivas) producen una consciencia intercultural, que incluye, naturalmente, la consciencia de la diversidad regional y social en ambos mundos, que se enriquece con la conciencia de una serie de culturas más amplia de la que conlleva la lengua materna y la segunda lengua, lo cual contribuye a ubicar ambas en su contexto. Además del conocimiento objetivo, la consciencia intercultural supone una toma de conciencia del modo en que aparece la comunidad desde la perspectiva de los demás, a menudo, en forma de estereotipos nacionales.

El MCERL, siguiendo a Canale (1983), Byram y Fleming (2001) y otros, opta por el hablante intercultural, en cuya configuración como tal deben desarrollarse una serie de habilidades y destrezas interculturales, definidas en su apartado 5.1.2.2:

* La capacidad de relacionar entre sí la cultura de origen y la cultura extranjera.

* La sensibilidad cultural y la capacidad de identificar y utilizar una variedad de estrategias para establecer contacto con personas de otras culturas.

* La capacidad de cumplir el papel de intermediario cultural entre la cultura propia y la cultura extranjera y de abordar con eficacia los malentendidos interculturales y las situaciones conflictivas.

* La capacidad de superar relaciones estereotipadas.

El aprendiente de una lengua extranjera, según el modelo de personalidad intercultural propuesto por el MCERL, desarrolla la interculturalidad en el proceso de adquisición de la lengua extranjera:

El alumno no adquiere dos formas de actuar y de comunicarse distintas y que no se relacionan, sino que se convierte en plurilingüe y desarrolla una interculturalidad. Las competencias lingüística y cultural respecto a cada lengua se modifican mediante el conocimiento de la otra lengua y contribuyen a crear una conciencia, unas destrezas y unas capacidades interculturales. Permiten que el individuo desarrolle una personalidad más rica y compleja, y mejoran la capacidad de aprendizaje posterior de lenguas y de apertura a nuevas experiencias culturales. Asimismo, capacitan también a los alumnos para mediar, a través de la interpretación y de la traducción, entre hablantes que no pueden comunicarse de forma directa en las dos lenguas implicadas. Naturalmente, hay un lugar para estas actividades (4.4.4) y competencias (5.1.1.3, 5.1.2.2. y 5.1.4), que diferencian al estudiante de la lengua del hablante nativo monolingüe.

Sin embargo, todas estas consideraciones siguen sin asegurarnos que a nivel comunicativo un aprendiente sea capaz de propiciar una comunicación intercultural.

La evolución del concepto de competencia comunicativa provocó la reformulación de la comunicación, medida ahora según sea o no eficaz. El problema surge cuando afirmamos que la comunicación intercultural (en la que se pondrán en práctica las des- 
trezas interculturales adquiridas por el aprendiente de una lengua extranjera) es un tipo especial de comunicación, que exige de unas pautas específicas y que está regulada por unos criterios algo distintos con respecto a aquélla. Alsina (1997) establece una serie de pautas o criterios para que la comunicación intercultural sea efectiva ${ }^{15}$ :

* Existencia de una lengua común

* Conocimiento de la cultura ajena y reconocimiento de la propia

* Eliminación de prejuicios

* Competencia empática

* Capacidad para metacomunicarse

* Producir una relación equilibrada

El primero de los criterios merece un comentario aparte, debido al tema estudiado en este artículo. La necesidad de que exista una lengua común es un requisito obvio, pero no por ello deja de implicar problemas dignos de mención: el hecho de adoptar una lengua (entiéndase, el inglés) como lingua franca (sobre todo en las lenguas de especialidad), puede, más que ayudar a la comprensión generalizada, provocar una ruptura entre el pensamiento y la forma de comprender la realidad exigida por la lengua adoptada ${ }^{16}$; razón por la cual es mucho más aconsejable en situaciones conversacionales interculturales elegir una lengua de comunicación más cercana a la cosmovisión lingüística y conceptual compartida (Balboni, 2000: 25-30).

El resto de criterios pueden ser relacionados con las destrezas indicadas por el MCERL, incluso aglutinados en torno a la competencia empática: facultad de ponerse en el lugar del otro y de reconocerse a uno mismo en el proceso comunicativo. Llama, no obstante la atención, la capacidad para metacomunicarse, puesto que la consecución de ésta podría bien ser considerada como cumplimiento de los demás: metacomunicarse es tener la capacidad de hablar de nuestros enunciados, de lo que dicen, de lo que quieren decir y de la reacción que esperan obtener por parte del otro.

La competencia intercultural, por tanto, sería la habilidad de un aprendiente de llevar a cabo situaciones de comunicación intercultural, por lo que en tales situaciones la competencia intercultural contendría dentro de sí la competencia comunicativa. De hecho, nuestro posicionamiento al respecto se basa en una concepción de la competencia intercultural como macrocompetencia ${ }^{17}$, cuyo radio de acción comprendería todo el resto de competencias (tanto las generales como las comunicativas). La competencia intercultural sería un estado evolucionado lógico de la competencia comunicativa. Al decir que se trata de una evolución lógica nos aproximamos a una concepción de la comunicación como sistema en continuo movimiento (proceso) dentro de un polisistema, también en evolución constante, que sería la sociedad ${ }^{18}$.

\footnotetext{
${ }^{15}$ Compárense con los criterios anteriormente citados de Woods (1998).

${ }^{16}$ Aún más drástica en aquellos casos en que la competencia intercultural esté indebidamente desarrollada.

${ }_{17}$ En parte, y basándonos en la capacidad para metacomunicarse, podríamos hablar de una metacompetencia.

${ }_{18}$ En otras áreas (y parcialmente en la metodología de la enseñanza y aprendizaje de lenguas extranjeras) podríamos encontrar formulaciones afines. Por ejemplo, en la Teoría de los Polisistemas
} 
El hecho de definir la competencia intercultural como macrocompetencia facilitaría la transversalización de la interculturalidad en los currículos, con lo que nos aproximaríamos más a la concepción de interculturalidad en tanto que estrategia de prevención que promueva la convivencia entre sociedades multiculturales. Situándola a este nivel, parecería justificado incluir la interculturalidad específicamente como competencia general en los currículos y planes de estudio tanto de enseñanzas no universitarias como universitarias. Así, la competencia intercultural sería, efectivamente, un enfoque, una conciencia en tanto que forma de actuar, de percibir, de decir, de comprender y de relacionarse. Su importancia en el aprendizaje de lenguas extranjeras es, pues, consecuencia de la imperiosa necesidad de una educación intercultural, pero quizá acentuada por ser precisamente en estas materias en las que, a través de la adquisición de la lengua como modelo de pensamiento y cosmovisión, el aprendiente tiene un contacto más directo con las habilidades que tendrá que desarrollar para poder ser un hablante intercultural.

Extraemos de lo anterior la conclusión, ampliamente respaldada ${ }^{19}$, de que la interculturalidad como modelo educativo implica el desarrollo de competencias en la percepción, cosmovisión, pensamiento y actuación. A este respecto, creemos ilustrativo reproducir la afirmación de Aguado (1998: 40):

La educación intercultural es un enfoque educativo basado en el respeto y valoración de la diversidad cultural, dirigido a todos y cada uno de los miembros de la sociedad en su conjunto, que propone un modelo de intervención, formal e informal, holístico, integrado, configurador de todas las dimensiones del proceso educativo en orden a lograr la igualdad de oportunidades/resultados, la superación del racismo en sus diversas manifestaciones, la comunicación y competencia interculturales.

Ahora bien, por su propia definición, la competencia intercultural exige no sólo de una adaptación de los currículos en los diferentes niveles educativos, sino también de una formación del profesor muy específica y difícilmente realizable. Hacia tal fin se orientan numerosas investigaciones recientes, en que se proponen modelos de adaptación curricular en interculturalidad más allá de la diversificación lingüística y su relación con el currículo que encontramos en el capítulo 8 del MCERL, así como otras que indagan en la formación del profesorado en interculturalidad. De entre todas las propuestas, destacan las estrategias diseñadas para desarrollar actitudes interculturales (Jordán, 1996: 107-121) y que presuponen un ejercicio tanto por parte del aprendiente como del profesorado. Como indican García Castaño et al. (1999), basándose en Jordán (1992), un modelo de formación del profesorado aceptado parece ser aquel en el que se contemplan:

* competencia cognitiva en teoría de la Educación Multicultural;

* conocimiento y compromiso respecto de una filosofía de la Educación Multicultural;

* conocimiento de las culturas en contacto;

* competencia pedagógica.

(entre otros, Even-Zohar) y la Lingüística Funcional Sistémica (entre otros, M.A.K. Halliday). La denominación de macrocompetencia tendría su base en el funcionamiento de las macroestructuras planteadas por Van Dijk (1980).

${ }_{19}$ Véase, entre otros, García Castaño, Granados Martínez y García-Cano Torrico (1999). 
Benamí Barros García, Galina M. Kharnásova $\quad$ La interculturalidad como macrocompetencia...

Este modelo, tal y como hacen ver García Castaño et alii (op.cit.), derivaría del de Gay (1986):

* Competencia cognitiva: adquisición de conceptos y planteamientos de la educación intercultural.

* Conocimiento y compromiso con una filosofía multicultural: la formación en las diferentes ideologías de la multiculturalidad y su acompañamiento de compromisos.

* Conocimiento de culturas en contacto: la adquisición de una mayor sensibilidad para percibir la diversidad como riqueza y no como déficit.

* Competencia pedagógica: adquisición de habilidades para identificar necesidades, poder interpretar libros de texto, elaborar adaptaciones curriculares, etc.

El problema que identificamos en estos modelos está principalmente relacionado con la poca frecuencia con que se señala «la necesidad de reestructurar los planes de estudio desde un enfoque intercultural, más allá de añadir contenidos obligatorios sobre esta temática» (Aguado, 2008: 282) y con la descontextualización de las tareas de formación del profesorado, que suelen realizarse fuera del Centro, factor que, de acuerdo con Jordán (1992), suele minar la eficiencia de tales actividades formativas:

La unidad de análisis para entender el desarrollo y formación del profesorado es la escuela, no solo considerada como un puesto de trabajo, sino también de formación compartida. Sólo si se produce un mayor perfeccionamiento del profesorado, un cambio de concepción en los curricula de los centros y un cambio en el clima de la institución escolar, la educación intercultural inclusiva cobra verdadero sentido. La escuela, así, se concibe como unidad de aprendizaje y trata de responder a las necesidades que esta va planteando, enfatizando la formación cooperativa y la reflexión compartida como claves para el desarrollo personal y profesional (Sales Ciges, 2010: 73).

Por todo lo expuesto, parece claro que el desarrollo de la (macro)competencia intercultural depende de una implicación global del sistema (desde las instituciones hasta los sujetos como integrantes de las comunidades multiculturales), tal y como ya dejaron claro García Martínez y Pérez Sánchez (1997). Por tanto,

$<\ldots>$ es preciso distanciarnos de cualquier propuesta que convierta a la escuela en un nicho aislado del resto de las instancias y espacios sociales; antes bien, esta manera de entender la realidad y las relaciones, o mejor, las interacciones entre concepciones diversas (en los contenidos y en las formas), debe trascender a toda la comunidad escolar. Es decir, un tal planteamiento requiere de la participación cooperativa de todos los agentes implicados (Camps, 1993; García, 1994) en las prácticas educativas, sean éstas escolares o extraescolares. En efecto, será demasiado pedir que la escuela, con sus propias fuerzas tuviera que eliminar todos los obstáculos que jalonan el camino hacia una sociedad intercultural. Para ello es imprescindible crear una cultura y unas estructuras de participación con poder real en la toma de decisiones, una actitud de tolerancia y el convencimiento de que lo 
distinto puede ser enriquecedor desde el momento en que pone en cuestión hasta los cimientos más aceptados, implícitos y dados por supuesto. Por este motivo, «La opción intercultural considera a la escuela como un lugar de encuentro entre culturas y como un ámbito privilegiado para el aprendizaje de las exigencias de la comunicación intercultural». (Abad, Cucó e Izquierdo, 1993: 50-51).

Resulta fácilmente deducible que las estrategias destinadas a la adquisición y desarrollo por parte del aprendiente de la competencia intercultural no serán en modo alguno meras actividades a realizar sólo en clase, sino que deberán contar con un grado de sincronización interdisciplinar que, a día de hoy, parece estar todavía lejano. No obstante, pueden proponerse ciertos enfoques que favorezcan la creación de una personalidad intercultural en el aprendiente, de cuya eficiencia dan buena muestra algunas experiencias llevadas a cabo en diferentes Centros $^{20}$. Por nuestra parte, creemos muy conveniente el progresivo desvío de las prácticas de interculturalidad en los Centros mediante programas compensatorios hacia un desarrollo integrado en el aula a través de programas globales, que incluyan a todos los partícipes del proceso de enseñanza y aprendizaje.

\section{El DESARRollo DE LA COMPETENCIA INTERCULTURAL: EXPECTATIVAS EN EL AULA DE LENGUA EXTRANJERA}

Creemos útil poner de relieve la relación que mantiene la competencia intercultural con todas las competencias definidas por el MCERL, no tanto con el fin de dar validez a la hipótesis de que en realidad se trate de una macrocompetencia, sino con el de subrayar las implicaciones metodológicas que pudiera conllevar a la hora de proponer actividades para su desarrollo en el aula.

Como es sabido, en su capítulo 5 el MCERL distingue entre competencias generales, divididas en cuatro saberes (saber, saber hacer, saber estar, saber aprender), y comunicativas (lingüísticas, sociolingüísticas, pragmáticas). La presencia de la competencia intercultural en casi todas ellas está de por sí justificada si atendemos a que, bien sea la consciencia intercultural, bien las destrezas interculturales, prácticamente todas las competencias tienen dentro de sí algún criterio relacionado con lo intercultural. En concreto, de entre las generales, los dos primeros saberes difícilmente podrán ser dominados si no se ha desarrollado en el aprendiente una competencia intercultural. La inclusión de la interculturalidad en las demás competencias y subcompetencias creemos que queda justificada si seguimos el esquema que proponen Byram, Gribkova y Starkey (2002) sobre los componentes de la competencia intercultural a desarrollar por un aprendiente ${ }^{21}$ :

* Los puntos de vista y perspectivas interculturales («saber ser»): apertura, capacidad para revisar la propia desconfianza frente a otras culturas y la fe en la de

20 Para un desarrollo más detallado de la cuestión, véase Aguado (2005).

21 Los enumeramos según la síntesis que realiza Paricio (2004). 
Benamí Barros García, Galina M. Kharnásova $\quad$ La interculturalidad como macrocompetencia...

uno mismo. Se trata de una voluntad de relativizar los propios valores, creencias y comportamientos, aceptando que no son los únicos posibles, y de aprender a considerarlos desde la perspectiva de una persona exterior, de alguien que tiene un conjunto de valores, creencias y comportamientos distintos.

* El conocimiento («saberes»): conocimiento de los distintos grupos sociales, de sus productos y de sus prácticas, tanto en el propio país como en el del interlocutor; conocimiento de las interacciones generales entre sociedades e individuos.

* Las capacidades de comparación, de interpretación y de establecer relaciones («saber comprender»): capacidad general para interpretar un documento o un acontecimiento vinculado a otra cultura, para explicarlo y ponerlo en relación con documentos o acontecimientos vinculados a la propia cultura.

* Las capacidades de descubrimiento y de interacción («saber aprender/ hacer»): capacidad para adquirir nuevos conocimientos sobre una cultura y unas prácticas culturales dadas, así como para manejar conocimientos, puntos de vista y capacidades sometiéndose a las normas de la comunicación y de la interacción en tiempo real.

* La visión crítica en el plano cultural («saber comprometerse»): capacidad para evaluar, de manera crítica, los puntos de vista, prácticas y productos del propio país y de las otras naciones y culturas.

Otra cuestión de relevancia es la de proponer actividades que, integradas en las competencias generales, objetivos y contenidos de los diferentes niveles educativos, sirvan efectivamente para desarrollar la competencia intercultural en el aula. En este sentido, conviene incidir en la necesidad de una formación del profesorado, labor que tendrá entre sus finalidades cambiar las pautas que regían la relación entre el profesor y el alumno, guiadas ahora por sus nuevos papeles en cuanto mediadores.

Como acertadamente destaca Paricio (2004), se pueden distinguir algunas tareas que tendrá que llevar a cabo el profesor de lenguas extranjeras en relación con los aprendientes de la mano de las propuestas de Byram, Gribkova y Starkey (2002):

* prepararlo para entablar relaciones con personas de otras culturas;

* favorecer que comprenda y acepte a esas personas diferentes como individuos que tienen distintos puntos de vista, valores y comportamientos;

* ayudarle a comprender el funcionamiento de las interacciones culturales;

* mostrarle que las identidades sociales son parte integrante de cualquier relación;

* demostrarle la influencia de la percepción que se tiene de los otros y de la visión que los otros tienen de uno mismo en el éxito de la comunicación;

* conducirle a saber más, por sí mismo, de las personas con las que se comunica;

* ayudarle a aprehender el carácter enriquecedor de este tipo de experiencias y relaciones.

El profesor tiene a su disposición gran número de métodos de trabajo y enfoques metodológicos para el desarrollo de actividades en el aula de lengua extranjera (entre otros: enfoque por tareas -task-based approach-, estudio de casos, simulaciones, pro- 
yectos, presentaciones orales, etcétera), que debe seguir poniendo en práctica, pero ha de ser capaz de elegir y definir el modelo metodológico idóneo para que se consiga una actividad que realmente favorezca y promueva el desarrollo de la competencia intercultural. Para ello, el profesor deberá evaluar desde un enfoque intercultural todas las cuestiones referentes a la elección de materiales y contenidos, adecuación de éstos y del propio modelo metodológico a los aprendientes y al contexto de aprendizaje, la coherencia con respecto a la programación didáctica general, la organización de materiales, tipología de actividades e, incluso, su propio comportamiento conversacional.

Con todo, la situación actual parece oscilar entre un optimismo que aprueba los esfuerzos institucionales por ampliar debidamente la presencia del componente cultural en los programas educativos europeos ${ }^{22}$ y un pesimismo en la práctica real de la interculturalidad en las aulas, basado en la supuesta evidencia de que ni los materiales, ni las guías docentes, ni las líneas de formación del profesorado están bien definidos para promover y desarrollar la interculturalidad ${ }^{23}$.

De hecho, no son pocos los investigadores que han puesto de manifiesto la necesidad de partir de un modelo teórico consolidado, como puede ser el Modelo de competencias interculturales transversales ${ }^{24}$.

Además, creemos plausible y oportuno comenzar a plantear las bases sobre las que cimentar un Modelo específico para la enseñanza y aprendizaje de lenguas extranjeras, basado en la macrocompetencia intercultural.

\section{El espacio comunicativo y el PluRilingüismo}

Como ya se ha puesto de relieve, gran parte de las expectativas de éxito de los estudios interculturales en su aplicación a las metodologías de enseñanza y aprendizaje de lenguas extranjeras pasa por la construcción de un modelo educativo capaz de optimizar las estrategias de adquisición y desarrollo de las diferentes competencias implicadas. En teoría, se pretende la elaboración de un modelo unificado, que abarcara el mayor espacio posible. Como es sabido, dentro del marco de la Unión Europea se están llevando a cabo numerosas investigaciones de gran valía en estos campos, aunque se parte también de la evidencia de que se trata de una ardua tarea, ya no sólo por las dificultades intrínsecas a los objetivos propuestos, sino también por el complejo engranaje que ha de ponerse en funcionamiento para que el modelo educativo pueda prosperar y ser realmente eficaz.

Probablemente, dentro de los últimos estudios y puestas en práctica sobre esta materia, la atención parece focalizarse en lo que siguiendo a Kramsch (1993) se denomina «el tercer espacio»; esto es, el nuevo espacio comunicativo en que se genera una cultura

22 Véase al respecto el informe elaborado por Eurydice (2001).

23 Véase la entrevista realizada a la profesora Teresa Aguado, accesible en http:// www.aulaintercultural.org/article.php3?id_article=741 [Consulta: 30 de julio de 2010].

${ }^{24}$ Para una explicación detallada de los modelos, véase Rodríguez Izquierdo (2008). Sobre la aplicación del Modelo de Competencias Interculturales Transversales (CIT), véase Aneas (2003, 2009). 
híbrida o intermedia entre las culturas que participan del acto de comunicación. Así, este concepto, recientemente propuesto como concepto nuclear, entre otros, por González Piñeiro, Guillén y Vez (2010), puede relacionarse con el enfoque holístico para el desarrollo de la competencia intercultural, que nos advierte de la necesidad de que el hablante no nativo (el buscado hablante intercultural referido anteriormente) se sitúe en un espacio intermedio entre la cultura propia que porta y transfiere por ser nativo hablante de una lengua y la cultura aprendida o de la lengua extranjera. Lo que parece claro es que los estudios sobre este nuevo espacio, el tercer espacio, han de determinar estrategias y modelos de optimización de los recursos, teorías y actividades para potenciar el desarrollo de las habilidades interculturales del hablante no nativo.

Ahora bien, la correcta administración de ese tercer espacio no será tarea fácil, precisamente por tener que controlar las oscilaciones propias de un estado intermedio que, en numerosas ocasiones, tenderá bien hacia la cultura de la lengua nativa, bien hacia la extranjera. Además, el tercer espacio no es ya un lugar estático de coexistencia de culturas o lenguas, sino que refiere a una realidad espacio-temporal de interrelaciones, por lo que no cualquier diálogo multicultural generará este tercer espacio; razón por la que su construcción se verá condicionada por la correcta superación de todos aquellos escollos inherentes a la comunicación intercultural ${ }^{25}$.

Así, el tercer espacio debe ser, por una parte, espacio sobre el que cimentar los nuevos modelos de enseñanza (no sólo) idiomática y, por otra, ámbito para el tratamiento de los posibles conflictos interculturales. De hecho, en recientes estudios se plantean estrategias para la resolución de conflictos del aula precisamente en este tercer espacio; por ejemplo, en temas como la transversalización del enfoque de género (Rebollo, 2006), lo que nos hace pensar que el tercer espacio no es sólo un concepto óptimo para la enseñanza, adquisición y desarrollo de las competencias plurilingües e interculturales, sino también para la transversalización de otros enfoques que andan en muchos aspectos encallados. También cabría la posibilidad de pensar, de un modo parecido al de Mohan y van Naerssen (1997) cuando proponía que ya no tenía mucho sentido hablar de la enseñanza y aprendizaje individual de cierta lengua, que las competencias transversales deben ser integradas por medio de un mismo enfoque, cuya base para la aplicación y puesta en práctica podría ser el tercer espacio.

El planteamiento de la educación idiomática en el tercer espacio nos permite ratificar lo que ya con los enfoques comunicativos se puso de manifiesto: el aprendizaje de una lengua ya no se debe basar en el conocimiento de un sistema lingüístico, sino en la concepción de que la lengua es una forma de transferencia de la visión del mundo lingüística y conceptual y que, por tanto, su dinamismo no sólo viene producido por factores lingüísticos, sino también por otros de diferente índole, dentro de los cuales los socioculturales tienen una importancia incuestionable. Aquella clásica advertencia de que la lengua debía ser conocida en su uso, ha de ser reformulada ahora bajo el prisma de los espacios comunicativos multiculturales:

${ }^{25}$ Para una sistematización de los posibles obstáculos para lograr el diálogo intercultural generador del tercer espacio, véase Vilà Baños (2008). 
Dominar una lengua va, ante todo, más allá de «conocer» sus formas: se trata de saber utilizarla para comunicar e interactuar, adecuando la lengua y sus variaciones a los contextos comunicativos plurales en los que está participando. $<\ldots>$ la competencia plurilingüe supera con mucho las habilidades monolingües. (Noguerol, 2008: 12).

El modelo educativo propuesto por Beacco y Byram (2007) ha cimentado la base para la elaboración de estrategias integradas para la enseñanza, aprendizaje y desarrollo de la competencia plurilingüe, elevando el plurilingüismo a la categoría de principio fundamental. Destaca de su modelo que para poder estructurarlo en torno a las habilidades lingüísticas necesarias, recurran a lo que, según nos parece, confirma la importancia que tuvieron los primeros inicios de la lingüística funcional sistémica en las investigaciones recientes en interculturalidad; esto es, al concepto de repertorio. Definido en los primeros trabajos dedicados a la competencia comunicativa como conjunto de recursos lingüísticos de que dispone cierta comunidad lingüística (Gumperz 1986: 20), ahora es visto desde una perspectiva más amplia, calificado de plurilingüe y entendido como consecuencia lógica y deseable de la adquisición, en un marco multicultural, de una competencia pluricultural (Beacco y Byram 2007: 35).

Parece evidente que los estudios sobre esta competencia serán los encargados de guiar los nuevos modelos educativos basados en el plurilingüismo, como dan fe algunas de las publicaciones más recientes en cuanto a los estudios interculturales en el ámbito de la enseñanza y adquisición de lenguas extranjeras. Entre otras, una de las tareas fundamentales a realizar sería la aplicación de los modelos plurilingües a los diferentes espacios educativos; empresa de no fácil sistematización y que en trabajos recientes ha sido perfectamente abordada, por ejemplo, en el marco de la Educación Secundaria (Baños 2005; Cots et alii 2010). Poco a poco empiezan a surgir, en parte alentadas por la implantación del Espacio Europeo de Enseñanza Superior, investigaciones y monografías dedicadas a la transversalización del enfoque intercultural en la Enseñanza Superior, sobre todo en el ámbito de la enseñanza y adquisición de lenguas extranjeras a través de métodos de aprendizaje basados en los, así llamados, «escenarios de comunicación» ${ }^{26}$.

$\mathrm{Y}$ es que la integración curricular de la competencia pluricultural o, desde una perspectiva sociológica, del enfoque intercultural, sigue constituyendo un reto, ya no sólo por las dificultades inherentes a la materia de que se trata, con una base terminológica y conceptual aún en desarrollo, sino también, y quizá sobre todo, por la necesaria implicación institucional para que se pueda llevar a cabo una adecuada adaptación del contexto educativo al plurilingüismo. Hablamos, como es sabido, de unos modelos educativos integrados no sólo en términos geopolíticos (por ejemplo, en el marco de la $\mathrm{UE}^{27}$ ), sino también en lo formativo, puesto que requieren un aparato institucional muy bien coordinado para poder dotarlos de eficacia. La formación previa de un profesorado que, en gran parte, puede desconocer las estrategias, mecanismos, actividades y utilidades del

${ }^{26}$ A este respecto, véase González Piñeiro, Guillén y Vez (2010).

${ }^{27}$ En cuanto a las condiciones y posibilidades para la elaboración de políticas lingüísticas capaces de integrar los modelos educativos plurilingües, véase Vez (2009). 
Benamí Barros García, Galina M. Kharnásova $\quad$ La interculturalidad como macrocompetencia...

plurilingüismo como modelo educativo, la instaurada y, a veces, oxidada concepción del aula como espacio educativo unidireccional e impermeable a los fenómenos y relaciones de interacción, la rigidez curricular y los problemas intrínsecos a cualquier tarea que pretenda la transversalización de enfoques integrados son algunas de las razones por las que parece clara la necesidad de hacer un esfuerzo conjunto para poder aplicar, poco a poco.

\section{A MOdo DE CONCLUSIÓN}

El enfoque intercultural exige de una predisposición de todos los actores de los que depende el proceso educativo, por lo que su efectiva implantación como modelo educativo (la educación intercultural) exige de no pocos cambios en la legislación, en la estructura de los Centros y, por supuesto, en la relación profesor-alumno. Esto se encamina hacia una concepción del aula como espacio de interrelación e intercambio y no como lugar estático, lo que atañe a la oposición entre enseñanza multicultural e intercultural.

Por su ampliamente justificada y asentada literatura, creemos que la competencia intercultural está ya en condiciones de ser planteada como competencia de las competencias, a lo que durante el presente estudio nos hemos referido con el término macrocompetencia. La aceptación de una competencia global podría, creemos, facilitar la elaboración de enfoques metodológicos capaces de optimizar el desarrollo de las competencias básicas en el aprendiente de lenguas extranjeras, ahora ya concebido como hablante intercultural y mediador.

La irrupción de la mediación intercultural en la enseñanza y adquisición de lenguas extranjeras obligaría a redefinir muchos de los baremos orientados hacia la evaluación del nivel de lengua adquirido por el alumnado, así como a concebir la comunicación en términos de eficacia y no de perfección gramatical. Este hecho tendría especial relevancia, y de hecho ya se está estudiando en la materia, en la enseñanza de lenguas extranjeras de especialidad.

Conscientes de que el desarrollo de la competencia intercultural sólo es posible si se orienta hacia tal fin la enseñanza de una lengua extranjera ya en sus primeras etapas de aprendizaje, pensamos que la labor a realizar por el profesorado de Educación Secundaria Obligatoria es decisiva $\mathrm{y}$, por tanto, exige de una preparación específica, así como de la ya mencionada implicación de todo lo que conforma el macrosistema educativo.

La interculturalidad, y por tanto su implantación en las programaciones didácticas de los diferentes niveles educativos, es enriquecedora para todos, en tanto que posibilita la mejor convivencia, desarrollo de habilidades empáticas, comprensión del mundo y autoconocimiento en una sociedad actual, caracterizada precisamente por la multiculturalidad. Por esta razón, y por todo lo expuesto, creemos que bien merece un esfuerzo de todos para saber aprender de ella, objetivo que sólo se cumplirá si logramos que el enfoque intercultural presida las relaciones humanas. Y no cabe ninguna duda de que para ello la educación ha de desempeñar la labor más importante. 


\section{Bibliografía}

Abad, L., Cucó, A. e Izquierdo, A. (1993). Inmigración, pluralismo y tolerancia. Madrid: PopularJCI.

Aguado, T. (2005). «La educación intercultural en la práctica escolar: investigación en el ámbito español», en XXI. Revista de Educación, 7: 43-52.

Aguado, T. (2008). «Proyecto INTER: una guía para aplicar la educación intercultural en la escuela», en Ipland, J. (coord.), La atención a la diversidad: diferentes miradas. Huelva: Hergué, 267-282.

Aneas, A. (2003). Competencias interculturales transversales en la empresa: un modelo para la detección de necesidades formativas. Barcelona: Universidad de Barcelona (tesis doctoral no publicada).

Aneas (2009). «Competencias interculturales transversales: su diagnóstico en equipos de trabajo de baja cualificación», en Revista de Investigación Educativa, 27, 1: 105-123.

Bachman, L. F. (1990). Fundamental considerations in language testing. Oxford: Oxford University Press.

Balboni, P. E. (2000). Le microlingue scientifico-professionali. Turín: UTET.

Bartolomé, M.; Cabrera, F.; Del Campo, J.; Espín, J. V.; Marín, M. A., y Rodríguez, M. (2002). Identidad y ciudadanía. Un reto a la educación intercultural. Madrid: Narcea.

Beacco, J.C. y Byram, M. (2007). From linguistic diversity to plurilingual education. Guide for the development of language education policies in Europe. Strasbourg: Language Policy Division.

Byram, M. (1997). Teaching and assessing intercultural competence. Clevendon: Multilingual Matters.

Byram, M. y Fleming, M. (2001). Perspectivas interculturales en el aprendizaje de idiomas: enfoques a través del teatro y la etnografía. Cambridge: Cambridge University Press.

Byram, M., Gribkova, B. y Starkey, H. (2002). Développer la dimension interculturelle de l'enseignement des langues. Une introduction à l'usage des enseignants. Strasbourg: Conseil de l'Europe.

Camps, V. (1993). Los valores de la educación. Madrid: Alauda.

Canale, M. (1983). «From communicative competence to communicative language pedagogy», en Richards, J. y Schmidt, R. (eds.), Language and communication. London: Longman, 2-27.

Canale, M. y Swain, M. (1980). «Theoretical Bases of Communicative Approches to Second Language Teaching and Testing», en Applied Linguistics, 1, 1: 1-47.

Celce-Murcia, M., Dörnyei, Z., Thurrell, S. (1995). «Communicative competence: A pedagogically motivated model with content specifications», en Issues in Applied Linguistics, 6: 5-35.

Cenoz, J. y Valencia, J. (eds.) (1996). La Competencia Pragmática: Elementos Lingüísticos y Psicosociales. Leioa: Universidad del País Vasco.

Cots, J.M., Ibarraran, A., Irún M., Lasagabaster, D., Llurda, E., Sierra, J.M. (2010). Plurilingüismo e interculturalidad en la escuela. Reflexiones y propuestas didácticas. Barcelona: Graó

Dubois, J. et al. (1994). Diccionario de lingüística. Madrid: Alianza Editorial.

EURYDICE (2001). La enseñanza de las Lenguas Extranjeras en el contexto escolar europeo. Madrid: MECD. Secretaría General de Educación y Formación Profesional. CIDE.

García Castaño, F.J. y Granados Martínez, A. (1999). Lecturas para educación intercultural. Madrid: Trotta.

García Castaño, F.J., Granados Martínez, A. y García-Cano Torrico, M. (1999). «De la educación multicultural e intercultural a la lengua y cultura de origen: reflexiones sobre el caso 
Benamí Barros García, Galina M. Kharnásova $\quad$ La interculturalidad como macrocompetencia...

español», en Franzé, A. (coord.), Lengua y cultura de origen. Madrid: Ediciones del Oriente y del Mediterráneo, 215-280.

García Martínez, A. (1994). «La formación intercultural del profesorado», en Anales de Pedagogía, 12-13: $65-80$.

García Martínez, A. y Pérez Sánchez, M. D. (1997). «La educación intercultural: una cita obligada en la formación del profesorado», en Revista Electrónica Interuniversitaria de Formación del Profesorado, 1, 0, accesible en http://www.uva.es/aufop/publica/actas/viii/edprima.htm. Consultado el 9 de agosto de 2010.

Gay, G. (1986). «Multicultural Teacher Education», en Banks, J.A. y Lynch, J. (eds.), Multicultural Education in Western Societies. London: Holt, Rinehart and Winston, 154-177.

Gómez Morón, R. (2004) «La competencia pragmático conversacional en los manuales de español como lengua extranjera: Breve revisión teórico práctica desde la teoría pragmática de la cortesía lingüística», en Rushtaller, S. y Lorenzo, F. (eds.). La competencia lingüística y comunicativa en el aprendizaje del ELE. Madrid: Edinumen, 141-153.

González Piñeiro, M., Guillén Díaz, C. y Vez, J. M. (2010). Didáctica de las Lenguas Modernas. Competencia Plurilingüe e Intercultural. Madrid: Síntesis.

Gumperz, J. (1982). Discourse strategies. Cambridge: Cambridge University Press.

Gumperz, J. J. y Hymes, D. (1986), «Introduction», en Gumperz, J. J. y Hymes, D. (eds.). Directions in Sociolinguistics. The Ethnography of Communication. Oxford, New York: Basil Blackwell, 1-25.

Hall, E.T. (1959). The Silent Language. New York: Doubleday.

Hymes, D. (1971). «Competence and performance in linguistic theory», en Huxley, R. y Ingram, E. (eds.), Acquisition of languages: Models and methods. New York: Academic Press, 323.

Hymes, D. (1972). "Models of the interaction of language and social life», en Gumperz, J. y Hymes D. (eds.), Directions in sociolinguistics: The ethnography of communication. New York: Holt, Rinehart and Winston, 35-71.

Israel, E. (1995). «Comunicació intercultural i construcció periodística de la diferencia», en Anàlisi, 18: $59-85$.

Jordan, J. A. (1992). L'educació multicultural. Barcelona: CEAC.

Jordán, J.A. (1996). Propuestas de educación intercultural para profesores. Barcelona: CEAC.

Kasper, G. y Rose, K. (2001). «Pragmatics in Language Teaching», en Rose, K. y Kasper, G. (eds.). Pragmatics in Language Teaching. Cambridge: CUP, 1-9.

Kramsch, C. (1993). Context and Culture in Language Teaching. Oxford: OUP.

Kramsch, C. (1998). Language and culture. Oxford: Oxford University Press.

Krashen, S.D. y Terrell, T.D. (1983). The natural approach: Language acquisition in the classroom. London: Prentice Hall Europe.

Mclaughlin, B. (1987). Theories of second language learning. London: Edward Arnold.

Mohan, B., y Van Naerssen, M. (1997). «Understanding Cause-Effect: Learning through language». Forum 35 (4), accesible en http://eca.state.gov/forum/vols/vol35/no4/p22.htm. Consultado el 17 de enero de 2011.

Noguerol, A. (2008). «El tratamiento integrado de las lenguas en el marco europeo», en Textos de Didáctica de la Lengua y de la Literatura, 47: 10-19.

Paricio, M. S. (2004). «Dimensión intercultural en la enseñanza de las lenguas». Revista Iberoamericana de Educación, accesible en http://europa.eu.int/eurlex/es/com/cnc/2003/ com2003 0449es01.pdf. Consultado el 15 de agosto de 2010. 
Pienemann, M. (1984). «Psychological Constraints on the Teachability of Languages», en Studies in Second Language Acquisition, 6/2: 186-212.

Pienemann, M. y Johnston, M. (1989). «An Acquisition-based Procedure for Second Language Assessment», en Australian Review of Applied Linguistics, 9, 1: 92-122.

Poyatos, F. (1994). La Comunicación no Verbal. Madrid: Itsmo.

Rebollo, M.A. (2006) (coord.). Género e interculturalidad: educar para la igualdad, Madrid: La Muralla.

Rodríguez Izquierdo, R. (2008). «Implicaciones para los planes de estudio de la competencia intercultural en la formación de profesores principiantes», en I Congreso Internacional sobre Profesorado Principiante e Inserción Profesional a la Docencia, Sevilla 25-27 de junio, accesible en http://prometeo.us.es/idea/congreso/pdf\%20comunicaciones/50.pdf $>$. Consultado el 15 de octubre de 2010.

Rodrigo Alsina, M. (1997). «Elementos para una comunicación intercultural», en Revista Cidob d'Afers Internacionals, 36 (mayo), 11-21.

Sáez Alonso, R. (2001). «La educación intercultural en el ámbito de la Educación para el Desarrollo Humano Sostenible», en Revista Complutense de Educación, 12, 2: 713-737.

Sales Ciges, M.A. (2010). «La formación intercultural inclusiva del profesorado: Hacia la transformación social», en Revista Latinoamericana de Inclusión Educativa, 4, 1: 65-82. http:/ /www.rinace.net/rlei/numeros/vol4num1/art3.pdf. Consulta: 12 de agosto de 2010.

Scheflen, A. E. (1984) Sistemas de Comunicación Humana. Barcelona: Kairós.

Van Dijk, T. A. (1980). Macroestructures. An Interdisciplinary Study of Global Structures in Discourse. Cognitions and Interaction. Hillsdale (Nueva Jersey): Lawrence Erlbaum.

Van Ek, J. (1986). Objectives for Foreign Language Learning (Vol I.). Estrasburgo: Council of Europe.

Vez, J. M. (2009). «Multilingual Education in Europe: Policy Developments», en Porta Linguarum, 12: 7-24.

Vilà Baños, R. (2008). La competencia comunicativa intercultural. Un estudio en el primer ciclo de la Educación secundaria Obligatoria. Madrid: MEC-CIDE.

Wierzbicka, A. (1997). Understanding Cultures Through Their Key Words: English, Russian, Polish, German, Japanese. New York: Oxford University Press.

Wierzbicka, A. (1999). Emotions Across Languages and Cultures: Diversity and universals. Cambridge: Cambridge University Press.

Woods, P. (1998). «El arte y la ciencia de enseñar», en Woods, P. Investigar el arte de la enseñanza. Barcelona: Paidos, 29-48.

Zanón, J. (2007). «Psicolingüística y didáctica de las lenguas: Una aproximación histórica y conceptual, en MarcoEle, 5, accesible en http://marcoele.com/num/5/02e3c099650f54607/ psicolinguistica.pdf. Consultado el 10 de agosto de 2010. 